\author{
MITSUBISHI ELECTRIC RESEARCH LABORATORIES
}

http://www.merl.com

\title{
NLOS Channel Identification and Mitigation in Ultra Wideband ToA-Based Wireless Sensor Networks
}

\author{
Nayef Alsindi, Chunjie Duan, Jinyun Zhang, Tsutomu Tsuboi
}

TR2009-006 March 2009

\begin{abstract}
Recently, Ultra Wideband (UWB) Time of Arrival(ToA)-based localization in Wireless Sensor Networks (WSNs)have received considerable attention. For a typical WSN operating in indoor environments, the localization performance can be degraded considerably due to the existence of non-line-of-sight (NLOS) channel conditions between the sensor nodes. In these channel conditions the ranging accuracy is degraded due to the attenuation and/or loss of the Direct Path (DP) signal which ultimately imposes a positive bias on the ToA-based distance estimation. As a result there is a need for robust algorithms that have the capability to identify and mitigate those NLOS ranging conditions. In this paper, we propose a novel, low complexity wireless channel condition estimation algorithm that identifies the condition of the channel. Based on the estimated ToA and Received Signal Strength (RSS) the algorithm identifies the channel condition which can be either LOS, NLOS-DP available, or NLOS-DP not available. A channel measurement campaign was conducted in an office environment and the measurement results confirms the validity of our algorithm. To integrate our channel condition estimation into the localization schemes, we propose two weight assignment schemes which generate either a s̈oft weightör a hard weight: Simulation results show that our estimator has robust performance with success rate of $85 \%$. The simulations also show that by taking advantage of the channel condition estimation, we are able to reduce the RMSE of the localization estimate by over $40 \%$.
\end{abstract}

IEEE Proceedings

This work may not be copied or reproduced in whole or in part for any commercial purpose. Permission to copy in whole or in part without payment of fee is granted for nonprofit educational and research purposes provided that all such whole or partial copies include the following: a notice that such copying is by permission of Mitsubishi Electric Research Laboratories, Inc.; an acknowledgment of the authors and individual contributions to the work; and all applicable portions of the copyright notice. Copying, reproduction, or republishing for any other purpose shall require a license with payment of fee to Mitsubishi Electric Research Laboratories, Inc. All rights reserved.

Copyright (C) Mitsubishi Electric Research Laboratories, Inc., 2009

201 Broadway, Cambridge, Massachusetts 02139 



\title{
NLOS Channel Identification and Mitigation in Ultra Wideband ToA-based Wireless Sensor Networks
}

\author{
Nayef Alsindi, Chunjie Duan, Member, IEEE, Jinyun Zhang, Fellow, IEEE, and Tsutomu Tsuboi
}

\begin{abstract}
Recently, Ultra Wideband (UWB) Time of Arrival (ToA)-based localization in Wireless Sensor Networks (WSNs) have received considerable attention. For a typical WSN operating in indoor environments, the localization performance can be degraded considerably due to the existence of non-line-ofsight (NLOS) channel conditions between the sensor nodes. In these channel conditions the ranging accuracy is degraded due to the attenuation and/or loss of the Direct Path (DP) signal which ultimately imposes a positive bias on the ToA-based distance estimation. As a result there is a need for robust algorithms that have the capability to identify and mitigate those NLOS ranging conditions. In this paper, we propose a novel, low complexity wireless channel condition estimation algorithm that identifies the condition of the channel. Based on the estimated ToA and Received Signal Strength (RSS) the algorithm identifies the channel condition which can be either LOS, NLOS - DP available, or NLOS - DP not available. A channel measurement campaign was conducted in an office environment and the measurement results confirms the validity of our algorithm. To integrate our channel condition estimation into the localization schemes, we propose two weight assignment schemes which generate either a "soft weight" or a "hard weight". Simulation results show that our estimator has robust performance with success rate of $85 \%$. The simulations also show that by taking advantage of the channel condition estimation, we are able to reduce the RMSE of the localization estimate by over $40 \%$.
\end{abstract}

Index Terms-Ultra Wideband, ToA-based ranging, NLOS, cooperative localization, wireless sensor networks.

\section{INTRODUCTION}

Cooperative localization in wireless sensor networks (WSNs) has the potential to enable object and personnel tracking applications in public, commercial and military services. Typical examples include inventory, equipment tracking in warehouses and manufacturing floors; patient and medical equipment tracking in hospitals; and first responder and solider tracking for fire-fighters and battle field troop monitoring/commanding. [1]. In addition, localization in WSN can greatly reduce the deployment and maintenance cost for such networks [2].

Time-of-Arrival (ToA) based ranging technique using UltraWideband radio has the potential to provide accuracy range

Manuscript received Jan 15, 2009. This work is supported by Renesas Technology Inc.

Nayef Alsindi is with the Mathworks Inc., Natick, MA. nayefalsindi@gmail.com. Chunjie Duan and Jinyun Zhang are with Mitsubishi Electric Research Labs, 201 Broadway, Cambridge, MA 02139 USA. \{duan, jzhang\}@merl.com. Tsutomo Tsuboi is with Renesas Technology Corp., Nippon Bldg. 2-6-2, Ote-machi, Chiyoda-ku, Tokyo 100-0004, Japan. tsuboi.tsutomu@ renesas.com measurement thanks to its broad bandwidth [4]. For a typical WSN connected with its nodes connected with $R$ links, the performance of localization is highly dependent on the condition of the propagation channel of these $R$ range measurements [10]. For range measurements under LOS channel conditions, the Direct Path (DP) is usually the strongest and UWB has been shown to offer excellent ranging accuracy [3], [5]. Unfortunately, range measurements in NLOS channel conditions face a more difficult challenge, since the DP is either detected but attenuated or completely blocked. In the former case, the ranging performance of UWB is slightly degraded since the DP is not the strongest path. In the latter situation the DP is severely attenuated and blocked due to harsh obstacles and the UWB ranging accuracy is degraded significantly. In this pathological condition, range estimates using the the strongest path or the detectable first arrival will experience a positive bias [1], [6]. Such a positive bias can be very large and therefore detrimental to the overall location accuracy especially if the localization algorithm uses all the available ranging measurements indiscriminately [1].

Two steps need to be taken to address this issue. The first is to identify correctly the channel conditions associated with the range measurements. The second is to mitigate the ranging errors, especially positive bias in NLOS channels, in the localization process. Recently, several NLOS identification and mitigation techniques have been proposed. In [7], the channel statistics (such as RMS delay spread) are used to identify NLOS links and a Weighted Least Squares (WLS) approach is used to mitigate the NLOS ranging errors. The paper, however, provides no physical justification since it was not based on actual channel measurements. Furthermore, the algorithm is computationally complex and energy inefficient as multiple range measurements are needed per link to build the channel statistics. In [8], frequency diversity is used to identify the direct-path blockage. Based on channel measurements in a typical indoor environment, the authors show that the variation of ToA estimation across frequency sub-bands has a positive correlation with the channel condition. This approach requires a frequency hopping capable RF front end and therefore the sensor nodes would have higher cost, complexity and power consumption. It is also difficult to isolate the frequency dependency of the antennas from the channel, which directly impacts the effectiveness of such approach. Authors in [9] provides an overview and performance comparison of several other NLOS detection algorithms for UWB localization, including running variance, confidence metric, channel statistics (delay 
spread) and change of SNR. The running variance algorithm essentially computes the variance of subsequent range estimates and compares it against a predetermined threshold to decide between LOS and NLOS. Confidence metric and delay spread are very similar to [7] in that the mobile user analyzes the multipath profile to reach a decision. Finally, change of SNR algorithm detects sudden change of SNR as indication of the channel moving from LOS to NLOS, or vise versa. A common weakness in these approaches is that the algorithms and performance analysis use "blind" channel classification of NLOS and therefore lacks physical soundness.

In this paper, we propose a low complexity algorithm for estimating the channel condition. The estimator combines the measured ToA and received signal strength (RSS) to compute the probability of each channel condition using empirical a priori statistical channel model information. Since RSS is already available in the receivers (for automatic gain control, acquisition and etc.), our algorithm requires little or no additional hardware, no additional transmissions and the computation complexity is low. The algorithm is validated by channel measurement results conducted in an office environment. We also propose a "soft" and a "hard" weight assignment schemes which can be easily integrated into the localization algorithms. Our simulation results show that the performance of the proposed estimator is robust and that the accuracy of localization algorithm is significantly improved.

The remainder of this paper is organized as follows: In Section II, we present the details of our ToA-RSS based channel condition estimation algorithm. The results of our channel measurement are reported and discussed in Section III, which serve as validation to our algorithm as they match our analysis and models. In Section IV, we first briefly discuss the general localization algorithms and then present the simulation results highlighting the performance of our algorithms. Section $\mathrm{V}$ concludes this paper.

\section{ToA-RSS BASED ChANNEL CONDITION Estimation}

As presented in existing literature, the channel condition of a radio link in a wireless network can be one of the followings [1], [6]:

1) LOS channel: the DP between the transmitting node and the receiving node is unobstructed and therefore at the receiver, the direct path contains significant portion of the total received signal power.

2) NLOS-DP channel: the LOS is obstructed but the DP signal between the transmitting node and the receiving node is attenuated but detected. Depending on the receiver sensitivity, some energy of the direct path can be received.

3) NLOS-NDP channel: the LOS is obstructed and the DP signal between the transmitting node and the receiving node is completely blocked and therefore no energy is detected at the receiver.

In this paper, these three channel conditions are denoted as $C_{0}, C_{1}, C_{2}$ respectively. In a realistic deployment environment, links in a WSN often are a mixture of all three channel conditions. Therefore, of the $R$ range measurements, a fraction of them will be corrupted by LOS errors while the others will be corrupted by NLOS-DP and NLOS-NDP errors.

The estimated ToA between the $i^{t h}$ and the $j^{t h}$ nodes can be expressed as $\hat{\tau}_{i j}=\tau_{i j}+\zeta_{i j}$, where $\tau_{i j}$ is the true ToA between the nodes and $\zeta_{i j}$ is the estimation error. The range estimation derived from the ToA estimation can be formally given as

$$
\hat{d}_{i j}=d_{i j}+z_{i j}+\epsilon_{i j}=d_{i j}+z_{i j}+ \begin{cases}0 & C_{0}, \\ \beta_{i j} & C_{1}, \\ \gamma_{i j} & C_{2} .\end{cases}
$$

where $d_{i j}=\sqrt{\left(x_{i}-x_{j}\right)^{2}+\left(y_{i}-y_{j}\right)^{2}}=\nu \tau_{i j}$ is the true distance between node $i$ and $j, \nu$ is the speed of signal propagation, $z_{i j}$ is a zero mean measurement error and $\epsilon_{i j}$ is the corrupting ranging error. Generally, $\gamma_{i j}>\beta_{i j} \gg\left|z_{i j}\right|$, i.e., the NLOS induced biases can be significantly larger. If not properly identified and treated, they will have detrimental effect on the localization accuracy.

In this paper, we propose a channel condition estimation scheme using both ToA-based range estimates and RSS measurements. The objective of our proposed scheme is as follows. Given a ToA-based range estimate, $\hat{d}$, and an RSS measurement, $\hat{r}_{R S S}$, we compute the conditional probability $p\left(C_{i} \mid \hat{d}, \hat{r}_{R S S}\right)$ for $i=1,2$ or 3 . The intuition is that a small ToA-based range estimation and a low RSS generally indicate some degree of NLOS blockage and possible ToA estimate degradation. Similarly large distance estimates and relatively high signal power would imply LOS channel conditions since that generally means smaller power decay.

Given that the RSS value is generally available to the receiving node for automatic gain control (AGC) loop, this information can be considered as free. Our approach only requires ToA-estimation, RSS measurements and a priori information regarding the distance-power relationship, which is generally known given the deployment environment.

We first state that the conditional probability $p\left(C_{i} \mid \hat{d}, \hat{r}_{R S S}\right)$ can be computed using the Bayes' equation,

$$
p\left(C_{i} \mid \hat{d}, \hat{r}_{R S S}\right)=\frac{f\left(\hat{r}_{R S S} \mid C_{i}, \hat{d}\right) p\left(C_{i} \mid \hat{d},\right)}{\sum_{k=0}^{2} f\left(\hat{r}_{R S S} \mid C_{k}, \hat{d}\right) p\left(C_{k} \mid \hat{d}\right)},
$$

where $f\left(\hat{r}_{R S S} \mid C_{i}, \hat{d}\right)$ is the distribution of the signal power for a given channel condition at a ToA estimated distance. $p\left(C_{i} \mid \hat{d}\right)$ is the probability of the channel condition given the ToA estimated distance which can be similarly obtained using the Bayes' equation,

$$
p\left(C_{i} \mid \hat{d}\right)=\frac{f\left(\hat{d} \mid C_{i}\right) p\left(C_{i}\right)}{\sum_{k=0}^{2} f\left(\hat{d} \mid C_{k}\right) p\left(C_{k}\right)},
$$

where $f\left(\hat{d} \mid C_{i}\right) p\left(C_{i}\right)$ is the distribution of the ToA-estimated distances given the channel condition and $p\left(C_{i}\right)$ is the probability of channel condition $C_{i}$. The $a$ priori information $f\left(\hat{r}_{R S S} \mid C_{i}, \hat{d}\right)$ can be obtained through channel measurements and modeling of the path loss $P L=P_{t}-r_{R S S}$, which describes the distance-power relationship. The path-loss model is typically given by

$$
P L=P_{0}+10 \alpha \log _{10}(d)+\chi
$$




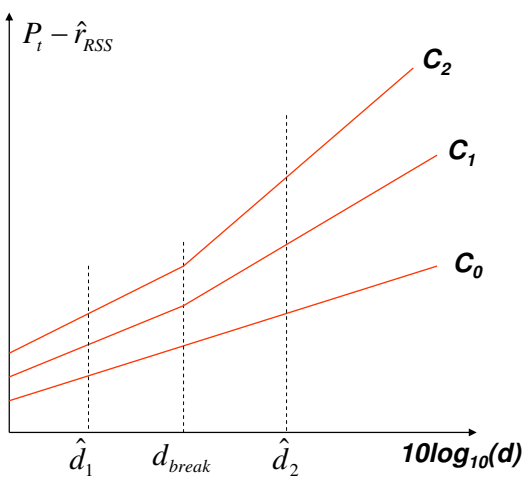

Fig. 1. Typical path loss behavior as a function of distance

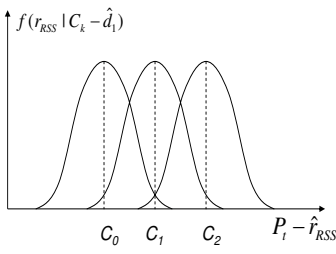

(a)

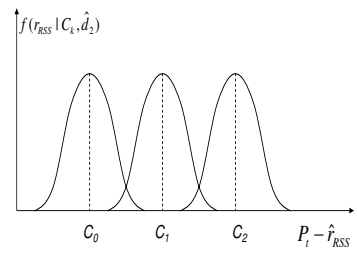

(b)
Fig. 2. Distribution of RSS at two different distance $d_{1}$ and $d_{2}$

where $P_{0}$ is the path-loss at a reference distance, typically 1 meter, $\alpha$ is the path-loss exponent and $\chi$ is the lognormally distributed shadow fading component. For NLOS channels, the path-loss can often fit to a two-piece model given as

$P L=\left\{\begin{array}{l}P_{0}+10 \alpha_{1} \log _{10}(d)+\chi_{1}, \\ P L\left(d_{\text {break }}\right)+10 \alpha_{2} \log _{10}\left(d / d_{\text {break }}\right)+\chi_{2},\end{array}\right.$ $d \leq d_{\text {break }}$
$d>d_{\text {break }}$

where $\alpha_{1}, \chi_{1}, \alpha_{2}$ and $\chi_{2}$ are the path-loss exponents and shadow fading before and after the break distance and their values vary in different channel conditions due to the different degrees of shadowing [1]. The break distance is a parameter determined by the environment. Figure 1 illustrates typical path-loss behaviors for the three different channel conditions. In LOS channels, there are no obstructions between the sensor nodes which results in lower distance-power decay. However, NLOS channels exhibit higher distance-power decay because of the obstacles between the sensors. The path-loss behavior between $C_{1}$ and $C_{2}$ can be further distinguished due to the fact that in the former the DP is always available, albeit attenuated, while the in the latter the blockage of the DP (and perhaps other shadowed MPCs) results in higher distance-power decay. This means that at a given estimated distance, it is possible to distinguish between the channel conditions using the RSS and the a priori path-loss models.

Naturally it would seem that channel identification using this technique might suffer at shorter distance compared to longer distance. As illustrated in Figure 2, at a close distance, e.g., $d_{1}$, the distributions are fairly close to each other, making the identification more difficult compared to the distribution at a longer distance, e.g., $d_{2}$. This situation is, however, somewhat alleviated for two reasons. First, at shorter distances the extent of the ranging error in the different conditions is not substantial. In other words, making a mistake in the identification between the three channel conditions at short distance has less significant impact on the localization since the ranging errors are not substantial. We will verify this claim through channel measurements and the distance-range error model in Section III. Secondly, when computing $p\left(C_{k} \mid \hat{d}, r_{R S S}\right)$ in (2), the PDFs $f\left(\hat{r}_{R S S} \mid C_{k}, \hat{d}\right)$ will be weighted by $p\left(C_{k} \mid \hat{d}\right)$, which is a function of $f\left(\hat{d} \mid C_{k}\right)$ and $p\left(C_{k}\right)$. For example, assume that the occurrence of the conditions is equiprobable, i.e., $p\left(C_{0}\right)=p\left(C_{1}\right)=p\left(C_{2}\right)$, then $f\left(\hat{d} \mid C_{0}\right)$ can be assumed as uniformly distributed between 0 and $d_{c}$, where $d_{c}$ is the communication range. $f\left(\hat{d} \mid C_{1}\right)$ and $f\left(\hat{d} \mid C_{2}\right)$ are distance dependent. Intuitively, $f\left(\hat{d} \mid C_{1}\right)$ is a monotonic decreasing function of $\hat{d}$ and $f\left(\hat{d} \mid C_{2}\right)$ is a monotonic increasing function of $\hat{d}$ since DP blockage becomes more likely with increasing distance in NLOS conditions.

The following is a set of distributions that satisfy these conditions:

$$
\begin{aligned}
& f\left(\hat{d} \mid C_{0}\right)= \begin{cases}\frac{1}{d_{c}} & d \leq d_{c} \\
0 & d>d_{c}\end{cases} \\
& f\left(\hat{d} \mid C_{1}\right)= \begin{cases}\frac{2\left(d_{c}-d\right)}{d_{c}^{2}} & d \leq d_{c} \\
0 & d>d_{c}\end{cases} \\
& f\left(\hat{d} \mid C_{2}\right)= \begin{cases}\frac{2 d}{d_{c}^{2}} & d \leq d_{c} \\
0 & d>d_{c}\end{cases}
\end{aligned}
$$

The simplicity of (5) and (6) can greatly reduce the overall computation complexity of the estimation algorithm based on (2) and (3). However, the validity of some these claims shall be verified not only by the theoretical analysis given above, but also through channel measurement results in a realistic indoor environment. The following section describes our channel measurement campaign in detail and shows that the measurement results matches our expectation (models).

\section{UWB Channel Measurement}

To validate the assumptions used in the Bayesian channel condition estimation algorithm proposed in Section II, we conducted frequency domain UWB indoor channel measurements. The measurements were conducted on the 7th floor of Mitsubishi Electric Research Labs, which is considered a typical office environment. 5 sets of measurement data were collected with a mixture of LOS and NLOS channels. Figure 3 illustrates the measurement locations. For each set of measurement, the transmitter was fixed at one location and the receiver was moved to different points, usually separated by $1 \mathrm{~m}$. Since the purpose of this measurement campaign is to develop robust NLOS identification algorithms, it was designed such that an extensive variety of channel response under different channel conditions were measured. For example, in one set of NLOS measurements the number of walls between the transmitter and receiver was limited to 12 walls in order to induce controlled DP attenuation with distance. In another set of NLOS measurement, the transmitter was fixed at the corner of the office area (location $\mathbf{B}$ in 
Figure 3) and the receiver was placed in different offices thereby the number of obstacles/walls increases with distance. This specifically provides situations where the obstacles and attenuation of the DP increases with distance. The collective measurement database was divided into LOS and NLOS. The NLOS measurement database was further divided into NLOSDP and NLOS-NDP at the post processing stage by examining if detectable energy is present at the expected ToA of DP signals.

The measurement setup is similar to what is described in [1], [6]. An Agilent E8363B vector network analyzer (VNA) is employed, which sweeps over the frequency range of 3-8 GHz. Frequency response of the channel, $H_{c h}$ or the S21 parameter is measured at 16001 sampling points, which translates into a frequency resolution of $\Delta f=312.5 \mathrm{~K} \mathrm{~Hz}$. To measure up to 15 meter antenna separation in NLOS channels with sufficient SNR, the VNA output is amplified by a $30 \mathrm{~dB}$ power amplifier (Mini-Circuit ZVE-8G+), which has the frequency range of 3$8 \mathrm{GHz}$. Both the transmitter and receiver antennas are $3.0 \mathrm{GHz}$ - $10 \mathrm{GHz}$, omni-directional UWB antennas designed at Mitsubishi Electric. The transmitter and receiver antenna heights were both fixed at 1.5 meters. Such a setup gives us an overall dynamic range of $120 \mathrm{~dB}$. The operation and data collection of the measurement setup is remotely controlled by a software written in LabView. This removes the impact of human motion in the vicinity of the measurement areas and guarantees static channels.

In ToA-based ranging, the discrete time domain channel impulse response (CIR), $h(n)$ is of more importance. The following are the steps taken to convert each frequency domain measurement to a time domain CIR.

- The channel frequency response is obtained by removing (calibrating) the frequency response of the measurement system (cables, amplifier, etc), $H_{\text {comp }}$, from the measured response $H_{\text {all }}$ or $H_{c h}(f)=H_{\text {all }} / H_{\text {comp }}$.

- Passing $H_{c h}$ through a windowing function, such as Hanning window $\left(W_{h n}\right)$ to reduce the noise side lobes: $H_{c h w}(f)=W_{h n}(f) * H_{c h}(f)$.

- Performing inverse Discrete Fourier transform (IDFT) or chirped $\mathrm{Z}$ transform (CZT) to obtain the time domain CIR: $h(t)=C Z T\left(H_{\text {chw }}(f)\right)$.

The $5 \mathrm{GHz}$ of bandwidth provides time-domain resolution of $\Delta t=0.2 \mathrm{~ns}$ and range resolution $\Delta d \approx 7 \mathrm{~cm}$. The expected DP ToA is computed by $\tau_{D P}=\frac{D_{\text {ant }}}{\nu}$, where $D_{\text {ant }}$ is the antenna separation, either measured directly or calculated from the coordinates.

For each measurement, the channel power-delay-profile (PDP) is generated directly from CIR as $|h(n)|^{2}$. Energy of the paths with $\hat{\tau}_{i}$ delay is the total signal energy within a $\Delta t$ window, centered at $\tau_{i}$. For example, the energy of DP, $\hat{\alpha}_{D P}$ is computed as the total energy within a window centered at $\tau_{D P}$, i.e., $\left[\tau_{D P}-\frac{\Delta t}{2}, \tau_{D P}+\frac{\Delta t}{2}\right]$, where $\Delta t$ is the sampling clock period of the receiver, e.g., $2 \mathrm{~ns}$ for $500 \mathrm{MHz}$.

Every measurement is sorted into the three channel conditions based on the properties of its DP components. In LOS measurements, DPs are unobstructed and therefore a significant portion of the total energy is contained in the DP bin, $\hat{\tau}_{D P}$. The NLOS measurements are further separated

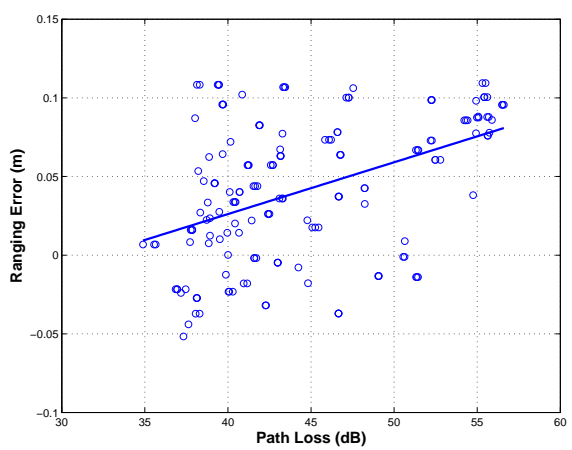

(a) NLOS-DP channels

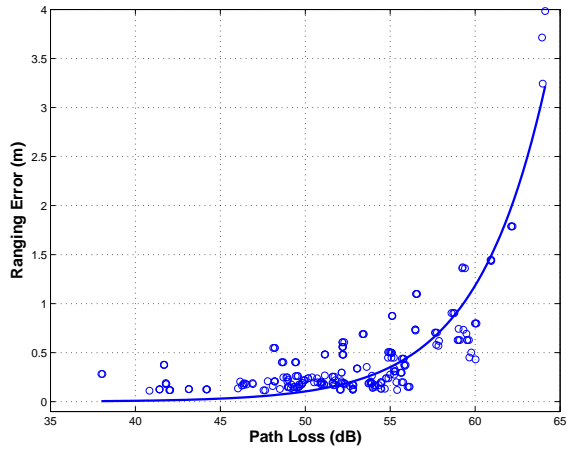

(b) NLOS-NDP channels

Fig. 4. Ranging error distribution vs. Path Loss in NLOS channels

into NLOS-DP and NLOS-NDP. If the energy within the first arrival bin, $\left[\tau_{D P}-\frac{\Delta t}{2}, \tau_{D P}+\frac{\Delta t}{2}\right]$, exceed a threshold (e.g., 10 $\mathrm{dB}$ above noise floor), DP is declared present and the channel is classified as NLOS-DP. Otherwise, the channel is considered NLOS-NDP.

For ToA-based estimation of UWB signals, accuracy is mainly dependant on the presence of the DPs. The measurement results show that in LOS scenarios, DPs are usually the strongest path and the ranging error has very little dependency on the total received power. The ranging error can be modeled as a Gaussian random variable with mean of $\mu=0.0033 \mathrm{~m}$ and standard deviation $\sigma=0.022$.

In NLOS-DP channels, the DP is not the strongest multipath component (MPC) even though it is detectable. In a bandlimited system, if we consider the power of the DP as the signal power then the SNR will be significantly lower than in the LOS channel conditions. The noise power here is composed of the additive Gaussian noise and energy from other MPCs close to the DP. This results in a larger ToA estimation error and higher dependency to the pass loss. The ranging error can again be modeled as a Gaussian distribution with a signal power dependent mean. Based on our measurement results, we have $\mu=0.06\left(P L_{d B}-35 d B\right)+0.01 m$ and standard deviation, $\sigma=0.039$.

In the case of NLOS-NDP channels, the direct path is completely absent. The expected ranging errors grow exponentially with the pathloss and can be modeled as

$$
\varrho=\kappa \exp \left(\varsigma \cdot P L_{d B}\right)+\varphi,
$$

Based on our measurements data, $\kappa=9.1 \times 10^{-7}, \varsigma=0.235$ 


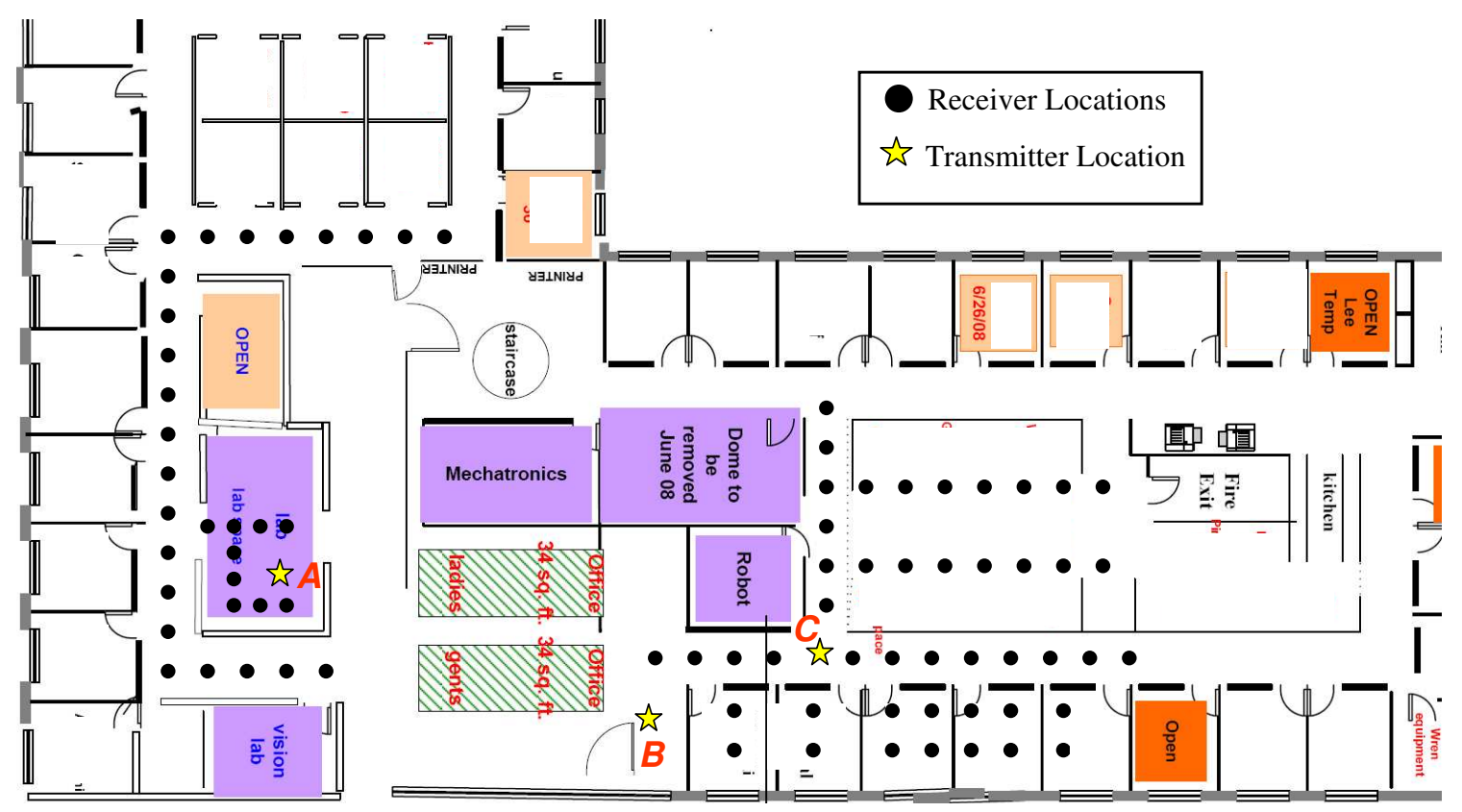

Fig. 3. Floor plan and measurement points

\begin{tabular}{|l|l|l|l|l|}
\hline \multirow{2}{*}{ Channel } & \multicolumn{2}{|c|}{$d \leq d_{\text {break }}$} & \multicolumn{2}{l|}{$d>d_{\text {break }}$} \\
\cline { 2 - 5 } & $\varsigma$ & $\chi$ & $\varsigma$ & $\chi$ \\
\hline LOS & 1.1 & 1.53 & 1.1 & 1.53 \\
NLOS-DP & 1.2 & 1.53 & 2.9 & 2.23 \\
NLOS-NDP & 1.3 & 2.04 & 3.2 & 3.05 \\
\hline
\end{tabular}

TABLE I

PATH LOSS PARAMETERS IN DIFFERENT CHANNEL CONDITIONS

and $\varphi$ is a Gaussian random variable with a standard deviation of $\sigma_{\varphi}=0.238$. Figure 4 plots the ranging error distributions in NLOS-DP and NLOS-NDP channels.

Our proposed channel condition estimation algorithm depends on the a priori information that describes the pathloss behavior in all three channel conditions. The empirical measurements and the corresponding pathloss models are shown in Figure 5 and the pathloss parameters obtained are provided in Table I. Path loss at 1-meter, $P_{0}$, is measured to be $33 \mathrm{~dB}$. The pathloss results and models show a clear separation between the LOS and NLOS channels. The pathloss in NLOSDP and NLOS-NDP diverge with increasing distance and it is clear from the figure that it is possible to distinguish between these two conditions.

Figure 6 plots the ranging errors in all channel conditions as a function of the antenna separation. Two observations can be made from the results. First of all, at short distances the error is well contained within 0.5 meters. This confirms our earlier assumption that at closer distance, missed detection of a channel condition will not results in significant localization performance degradation. The second is that the severity of ranging error at longer distances can be significant, emphasize the importance of correct channel condition estimation/detection.

We also reason that the distribution of NLOS-DP, $f\left(d \mid C_{1}\right)$, should be a monotonically decreasing function of the antenna

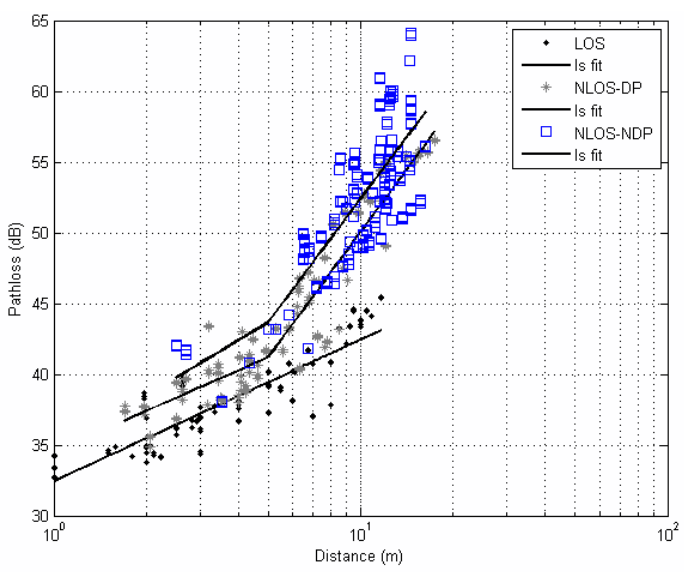

Fig. 5. Measured Pathloss for the three channel conditions. LOS, NLOS-DP and NLOS -NDP

separation and $f\left(d \mid C_{2}\right)$ a monotonically increasing function. This is confirmed by examining Figure 7, which shows the histogram of the measured distances in NLOS-DP and NLOSNDP. The distributions loosely fit the linear model of $f\left(d \mid C_{1}\right)$ and $f\left(d \mid C_{2}\right)$ given in (6).

\section{WSN LOCALIZATION AND EXPERIMENTAL RESULTS}

The ultimate goal of providing a reliable channel condition estimation/detection algorithm is to facilitate the localization algorithm design for improved accuracy. Node localization can be implemented in both traditional networks and ad-hoc sensor networks. In traditional networks, ranging measurement is only performed between the mobile terminal (MT) and the reference point/base station (BS). In WSNs, ranging information is exchanged between sensor nodes and anchor nodes in order to achieve localization for the entire network. The set 


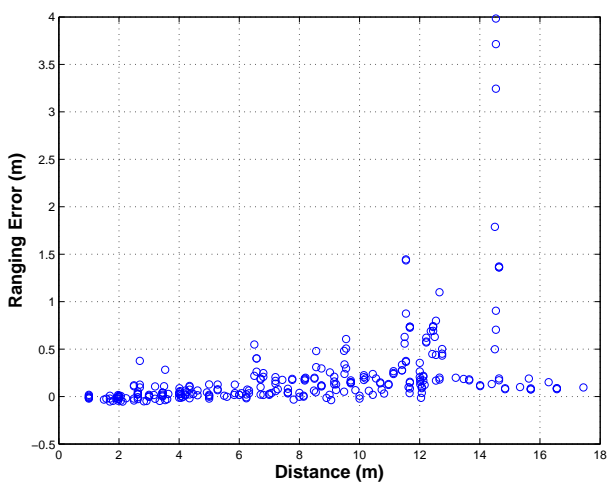

Fig. 6. Ranging errors vs. Antenna separation

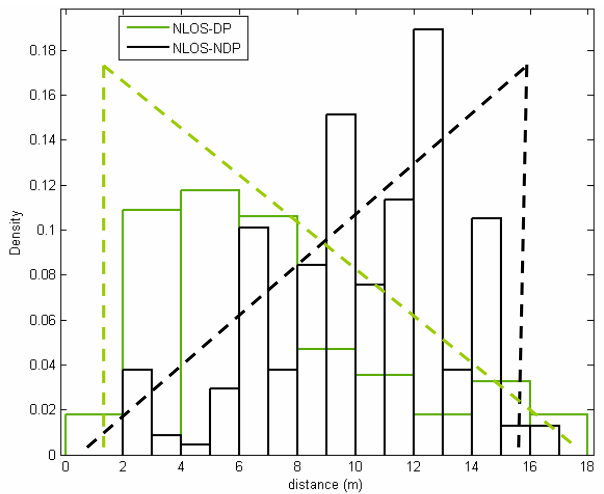

Fig. 7. Distribution of in NLOS conditions

of nodes with a priori location information, $A$, are referred to as "anchor nodes". The nodes with unknown location are referred to as "sensor nodes" hereafter and the set is denoted as $S$. The localization process is to estimate the location of all nodes in $S$ based on all or some of the available range estimates between nodes in both $S$ and $A$. In either networks, the impact of corrupted or biased ToA-based ranging have shown in [10] to yield significant localization error and many instances useless location information. We will show that when our identification and mitigation algorithm is integrated in both types of networks, substantial performance gains can be achieved.

The performance of localization can be measured quantitatively with Root Mean Square Error (RMSE) of the node location, given by

$$
\operatorname{RMSE}(\theta)=\sqrt{\sum_{i \in S}\left(x_{i}-\hat{x}_{i}\right)^{2}+\left(y_{i}-\hat{y}_{i}\right)^{2}},
$$

where $\left(x_{i}, y_{i}\right)$ is the true coordinate of node $i$ and $\left(\hat{x}_{i}, \hat{y}_{i}\right)$ the estimated coordinate. Without information about the corresponding channel conditions of the range estimates, the localization algorithms treat all range values equally. In such case, large ranging errors of the NLOS links often degrade the overall RMSE. If the channel condition estimate is available to the the localization process, algorithms can be designed to mitigate the NLOS-induced ranging error. Two approaches of using the output of channel condition estimator are described as follows.

1) A "hard" decision on the channel condition can be carried out by comparing $p\left(C \mid \hat{d}, r_{R S S}\right)$ for all three conditions and selecting the condition which maximizes $p\left(C \mid \hat{d}, r_{R S S}\right)$, as

$$
C_{k}=\arg \max _{k} p\left(C_{k} \mid \hat{d}, \hat{R S S}\right)
$$

The identification can be incorporated into a NLOS mitigating localization algorithm, such as WLS localization algorithm. A "hard" weight, $w$, is assigned to each link based on its identified channel condition, $C_{k}$.

2) Alternatively, a "soft" weight assignment scheme can be used. The weight is computed as follows

$$
w=\frac{\sum_{k=0}^{2} G_{k} p\left(C_{k} \mid \hat{d}, r_{\hat{R S S}}\right)}{\sum_{k=0}^{2} p\left(C_{k} \mid \hat{d}, r_{R S S}\right)}
$$

where $G_{0}, G_{1}$ and $G_{2}$ are hard weights that we select for the channel conditions LOS, NLOS-DP, and NLOSNDP respectively and satisfies $G_{0}>G_{1}>G_{2}$. In our simulations, we choose $G_{0}=10, G_{1}=1$ and $G_{2}=0.1$.

Once the weight of each link is determined, a WLS solution of node $i,(i \in S)$ location $\hat{\theta}=\left[\hat{x}_{i}, \hat{y}_{i}\right]$ is the solution that minimizes the cost function given by [11]

$$
E[\hat{\theta}]=[\hat{d}-F(\hat{\theta})]^{T} W[\hat{d}-F(\hat{\theta})],
$$

where $\hat{d}$ is the range measurement vector to the anchor nodes and $F(\hat{\theta})$ is the vector of estimated distance given by

$$
F(\hat{\theta})=\left[\begin{array}{c}
\sqrt{\left(\hat{x}_{i}-x_{1}^{A}\right)^{2}+\left(\hat{y}_{i}-y_{1}^{A}\right)^{2}} \\
\cdots \\
\sqrt{\left(\hat{x}_{i}-x_{M}^{A}\right)^{2}+\left(\hat{y}_{i}-y_{M}^{A}\right)^{2}}
\end{array}\right]
$$

where $\left(x_{m}^{A}, y_{m}^{A}\right)$ are the known coordinates of the $m^{\text {th }}$ anchor node, $(m \in A)$, and $W$ is a diagonal matrix, $W=$ $\operatorname{diag}\left(w_{k}^{1}, \cdots, w_{k}^{M}\right)$, with the diagonal element being the "hard weights" obtained from (8) or "soft weight" from (10).

We first simulate the performance of the algorithm in a traditional network consists of a single MT and 4 BSs. The MT is deployed randomly in a $15 m \times 15 m$ grid. Four BSs are placed at the corners of the grid. For each link between the MT and BSs, the channel conditions are generated randomly with equal probability, $p\left(C_{0}\right)=p\left(C_{1}\right)=p\left(C_{2}\right)$ and the RSS and ToA based range measurement are then generated randomly according to the channel models developed in section III. Based on the RSS and ToA measurements, the channel condition is then estimated according to the algorithm and a "soft" weight is used in node localization. In this experiment we ran 100 simulations and 100 node locations per simulation, a total of 40,000 simulated range measurements. The localization is simulated using both the channel-blind LS algorithm and our our proposed NLOS identification/mitigation algorithm.

As a first observation, our simulation results show that a "hard decision" channel identifier based on (8) has a success detection rate of $85 \%$, indicating the robustness of our NLOS identification approach. The second observation, drawn by comparing the position RMSE CDFs of two different 


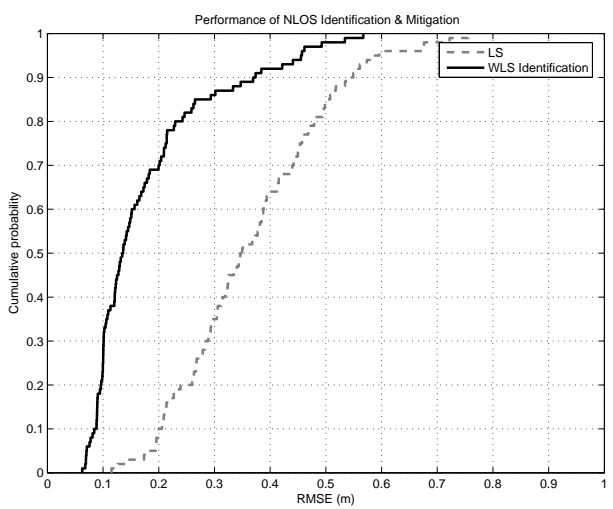

Fig. 8. CDF of location RMSE using WLS localization algorithm against an LS localization algorithm

algorithms (Figure 8), is that localization with our channel condition estimation and identification algorithm reduces the RMSE substantially. For instance, RMSE at $80 \%$ confidence level is reduced from $48 \mathrm{~cm}$ to $23 \mathrm{~cm}$ when our channel condition estimation and mitigation is used.

In the second set of experiments, we simulate the performance of our proposed algorithm in a WSN. The algorithm used in the simulation is the iterative distributed localization described in [12], [13]. In this type of cooperative localization the network is composed of blind sensor nodes and small fraction of anchor nodes. In each iteration, sensor nodes range to first-hop neighbors and if they hear from at least 3 anchor nodes then they perform multilateration and estimate their positions. The newly localized nodes then transform to anchor nodes and aide the remaining sensor nodes in the iterative localization process. Once again, both the channel-blind LS and our proposed WLS identification and mitigation algorithm are simulated. The setup of the simulation consists of 12 anchor nodes and 80 sensor nodes. The sensor nodes are randomly deployed in a circle with $12.5 \mathrm{~m}$ radius and the anchor nodes are deployed at the outer edge the sensor field. The angular deployment of the anchor nodes is uniformly distributed, while the radial deployment is uniformly distributed between 12.5 and $15 \mathrm{~m}$. Again the ToA and RSS ranging is obtained through the developed empirical models from section III.

Figure 9 gives one sample of the localization results. The actual node locations (represented by ·), estimated locations using the LS algorithm ( $\square$ ) and estimated locations using our proposed algorithm $(\times)$ are all plotted in the same figure. Anchor nodes are marked as $\oplus$. The LS-based algorithm experiences substantial position estimation degradation towards the edge of the network. In comparison, our proposed algorithm shows substantial position estimation improvements. Figure 10 plots the respective CDFs of the RMSE for both the LS and WLS-based localizations in WSN for a total of 400 realizations. Using our proposed algorithm, we are able to achieve 0.85 meter RMSE at $80 \%$ confidence level, as opposed to over $2 \mathrm{~m}$ for the conventional LS based algorithm, a $58 \%$ improvement.

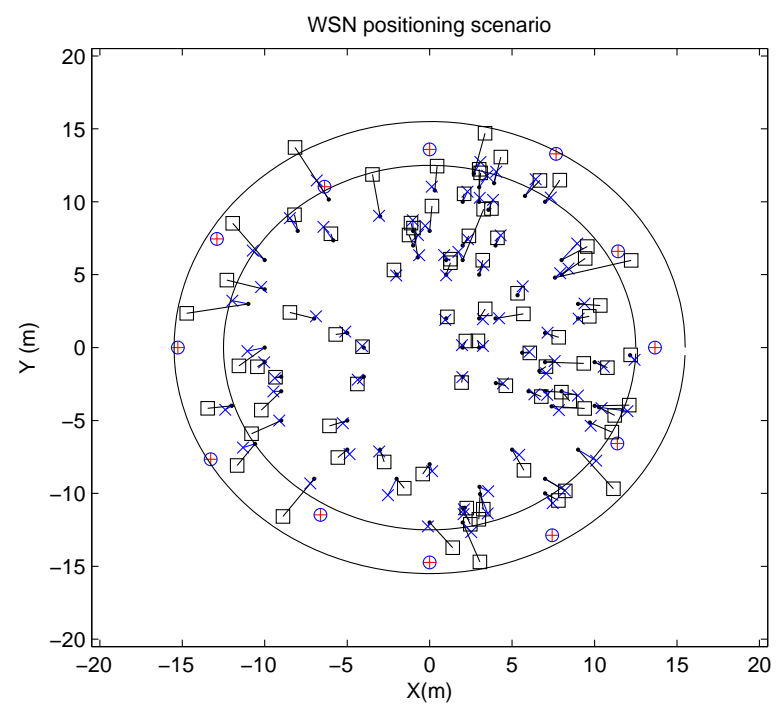

Fig. 9. Simulated WSN localization results

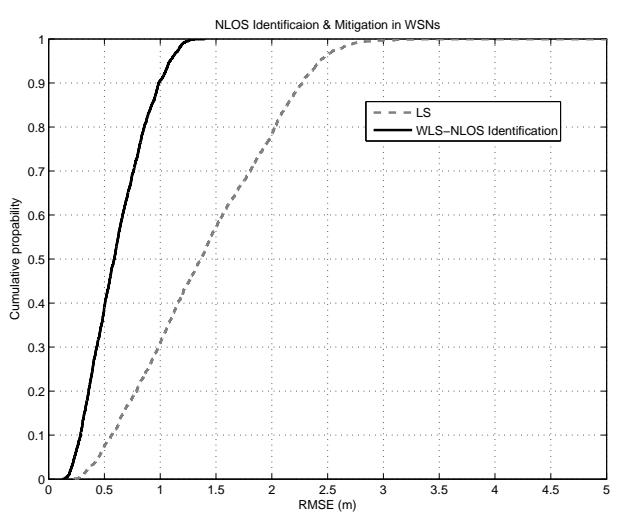

Fig. 10. CDF of location RMSE

\section{CONCLUSION}

UWB technology allows precision wireless localization and therefore provides a viable technology for enabling reliable and robust location based services in WSN applications. A major challenge facing distance/range estimation is the bias presented in NLOS channels and as a result, correctly identifying such channel condition is critical to the overall localization performance. This paper presents a low cost, low complexity channel condition estimation algorithm which utilizes both estimated ToA and received signal strength in addition to a priori channel model information. The validity of our algorithm is supported by the channel measurement conducted in an indoor environment. Further, the robustness of our estimator has been confirmed with both measurement and simulation data. We also proposed two weight assignment schemes and show through simulation results that by incorporating our channel condition estimation into localization processes, we are able to achieve substantial performance improvement in both traditional and sensor networks. 


\section{ACKNOWLEDGEMENTS}

The authors would like to thank Simarjit Anand for developing the LabView channel measurement control software, and Dr. Andreas F. Molisch for insightful discussions about the channel measurement and modeling. This project is partially funded by Renesas Technology Corporation.

\section{REFERENCES}

[1] K. Pahlavan, X. Li and J. Makela, "Indoor geolocation science and technology," IEEE Communications Magazine, vol. 40, no. 2, pp. 112118, Feb. 2002

[2] N. Patwari, J.N. Ash, S. Kyperountas, A. O. Hero, R. L. Moses and N. S. Correal, "Locating the nodes: cooperative localization in wireless sensor networks", IEEE Signal Processing Magazine, vol. 22, no. 4, pp. 54-69, July 2005.

[3] J.-Y. Lee and R.A. Scholtz, "Ranging in a dense multipath environment using an UWB radio link", IEEE Transactions on Selected Areas in Communications, vol. 20, no. 9, pp.1677-1683, Dec. 2002.

[4] S. Gezici, Z. Tian, G. Biannakis, H. Kobayashi, A. F. Molisch, H. V. Poor and Z. Sahinoglu, "Localization via Ultra-Wideband Radios", IEEE Signal Processing Magazine, vol. 22, no. 4, pp.70-84, July, 2005

[5] K. Pahlavan, F.O. Akgul, M. Heidari, A. Hatami, J.M. Elwell and R.D. Tingley, "Indoor geolocation in the absence of the direct path", IEEE Wireless Communications Magazine, vol. 13, no. 6, pp. 50-58, Dec. 2006.

[6] N. Alsindi, B. Alavi, K. Pahlavan, "Spatial characteristics of UWB TOA-based ranging in indoor multipath environments" in Proc. of IEEE International Symposium on Personal Indoor and Mobile Radio Communications (PIMRC) '07, Athens, Greece, pp. 1-6, Sept. 2007.

[7] I. Guvenc, C.-C. Chong and F. Watanabe, "NLOS identification and mitigation for UWB localization systems" IEEE Wireless Communications and Networking Conference (WCNC) '07, pp. 1571-1576, Hong Kong, China, March 11-15 2007.

[8] N. Alsindi, M. Heidari, K. Pahlavan, "Blockage identification in indoor UWB ranging using multiband OFDM signals", in Proceedings of IEEE Wireless Communications and Networking Conference, pp. 3231-3236, Las Vegas, Nevada, April 2008.

[9] J. Schroeder, S. Galler, K. Kyamakya and K. Jobmann, ”NLOS detection algorithms for Ultra-wideband localization", in Proceedings of 4th Workshop on Positioning, Navigation and Communication, pp. 159-166, March, 2007.

[10] N. Alsindi, K. Pahlavan, "Cooperative localization bounds for indoor ultra wideband sensor networks", EURASIP ASP special issue on Cooperative Localization in Wireless Ad Hoc and Sensor Networks, vol. 2008, article ID 852509, pp. 1-13, April 2008.

[11] S.M. Kay, Fundamentals of Statistical Signal Processing: Estimation Theory, Englewood Cliffs, NJ: Prentice-Hall, 1993.

[12] J. Albowicz, A. Chen and L. Zhang, ”Recursive position estimation in sensor networks", in Proc. of 2001 IEEE International Conference on Network Protocols (ICNP'01), Riverside, CA, pp. 35-41, Nov. 2001.

[13] N. Alsindi, "Indoor cooperative localization for Ultra Wideband Wireless Sensor Networks", Ph.D. Dissertation, May 2008. 